Copyright 1910. By A.I.E.E.

\title{
THE AUTOMATIC TELEPHONE IN RELATION TO CITY SERVICE.
}

BY ARTHUR BESSEY SMITH

The telephone requirements of a large city differ in certain essential points from those of a city of small or moderate size. The chief of these are as follows:

Measured service.

Private branch exchange service.

Interborough service.

Although these exist to some extent in smaller places, they are peculiarly important considerations in the telephone service of a large and congested city. They are practically the conditions which had to be met by the automatic telephone exchanges installed in Oakland and San Francisco, California. For this reason it is felt that a statement of the chief engineering problems of these cities and how they were solved will be of general interest.

The Setting. The interest in the region of the Golden Gate centers about two cities, San Francisco and Oakland. The former is a thriving seaport of about 350,000 inhabitants. It occupies the rough, hilly, northern end of the small peninsula (see Fig. 1) which separates the southern end of San Francisco bay from the Pacific ocean. The rather sandy region south of the city is thinly populated. The north peninsula across the Golden Gate is more thickly settled. To the eastward across the bay lies the city of Oakland, in the pleasant region between the Berkeley hills and the water. Oakland has a population of over 250,000 while Berkeley, Alameda and other smaller cities have about 60,000 inhabitants. The total population of the region reaches well up to three-quarters of a million.

San Francisco is a cosmopolitan city. There are Chinese, 
Italian and Latin quarters, each with its distinctive population and language. The wharves lie along the eastern edge of the city, with a great wholesale and manufacturing district south of the business center. Although a large amount of manufacturing is carried on, the harbor and shipping facilities have located the city and made it what it is.

Oakland has had a rapid growth since the earthquake across

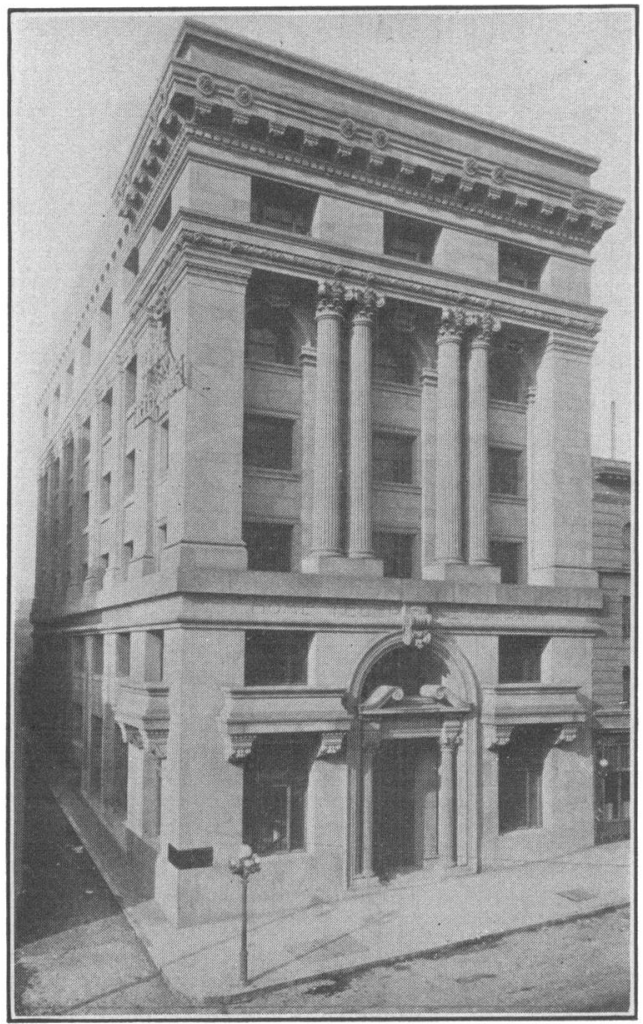

Main office, San Francisco

the bay and is second only to San Francisco in business importance. The cities are very closely bound together by business relations and also because so many business men of San Francisco live in Oakland. North of Oakland is Berkeley, the seat of the State University of California. Oakland also has considerable Chinese population, enough to warrant the serious attention of the telephone engineer. 
The Problem Stated. The requirements to be considered in designing a telephone system for this region may be briefly stated as follows:

1. A system which shall give satisfactory telephone transmission between all points in the whole territory.

2. A system which provides satisfactory accessory conditions such as ease, rapidity and accuracy of completing and controlling connections, methods of charging for service and means for discrimination in the same.

The consideration of these general requirements as applied to the case in hand causes their expansion into ten conditions, viz.,

1. Quiet, clear transmission over all talking circuits.

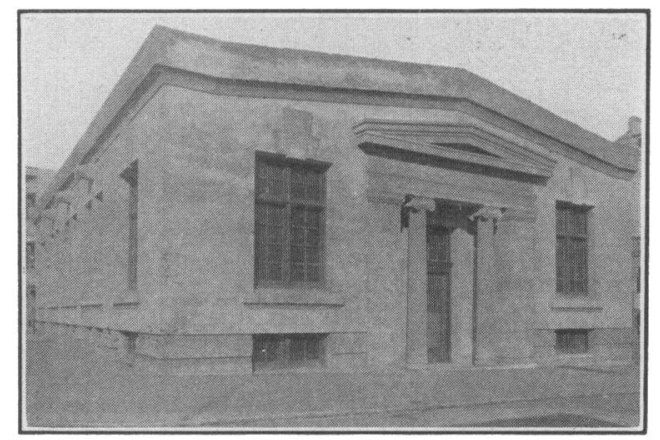

South office, San Francisco

2. Easy, quick and accurate completion of local connections within each exchange district.

3. Provision for the cosmopolitan nature of the population especially regarding the diversity of languages.

4. Measured service.

5. Free service on calls to certain classes of stations.

6. Private branch exchange business.

7 Quick and accurate completion of calls between exchange districts especially between San Francisco and Oakland.

8. Accuracy in charging the accounts of credit toll users.

9. Credit and cash toll work between exchanges.

10. Provision for the harmonious mutual working of different types of apparatus used in the several exchanges.

A Bit of History. In the Spring of 1905 the interest of San Francisco citizens was aroused by a small exhibit of the auto- 
matic system. This exhibit was, however, destroyed by the earthquake and fire in the spring of 1906 . While the city was recovering from the effects of the disaster the attention of constructionists was diverted to Oakland where an automatic system was installed and completed in May, 1907. It is of the three-wire trunk release common battery type, with Keith type line switches. Three offices are in service, Main East and Berkeley, with two district stations connected to the Main office.

Work was again taken up in San Francisco with the result that a system was installed in 1909. By the time the San Fran-

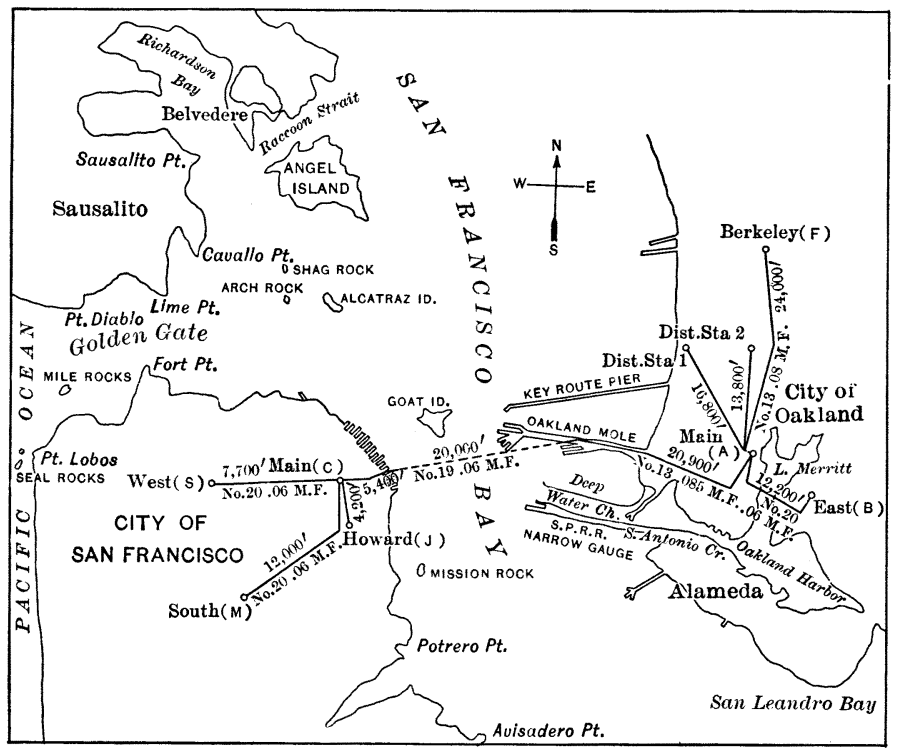

Fig. 1-Map of San Francisco and Oakland

cisco order was placed the two-wire automatic equipment had been perfected and was accordingly used. Four offices are at present operating and are designated as Main, Howard, South and West.

\section{Local Exchange Apparatus.}

Oakland. The locations of the offices in Oakland are shown in Fig. 1. More extended and detailed descriptions of local exchange apparatus have been given from time to time, hence my references will be confined to recalling the types and chief functions of the several switches, in order that the subjects discussed may be more clear. 
Each subscriber's line in any office terminates in a line switch (Fig. 2). These line switches are grouped in units (see Figs. 3 and 4) of from 50 to 100 each. All the line switches in a unit have access to a group of ten trunk lines. The general arrangement of switches is shown in Fig. 5. When any subscriber initiates a call, his line is automatically connected to an idle trunk line, $a$. This trunk is preselected, so that there is no loss of time, the connection being practically instantaneous. The various groups of ten trunks each, which come from the banks of the line switch units, terminate in first selector switches.

To the first or bottom level of bank contacts in all first se-

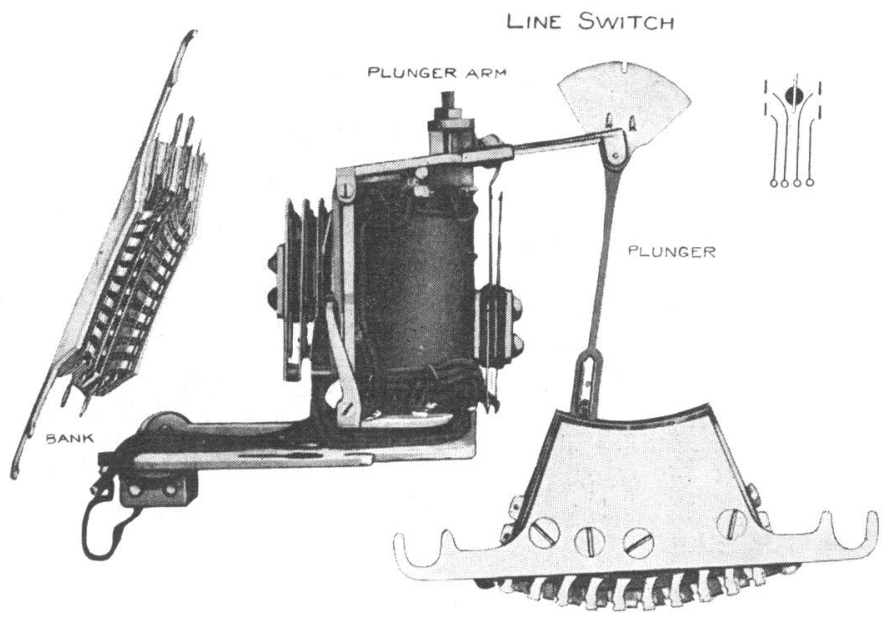

FIG. 2

lectors in Oakland are attached trunk lines leading to the Main office. The second level has the trunks leading to the East office and the third level those to the Berkeley office. Taking the East office for example, the trunks from the second level are termed local trunks, $b$, since they run to second selectors in the same office. Those from the first and third levels, however, are outgoing trunks, $c$, for they run to the other offices, Main and Berkeley. These outgoing trunks go through repeaters, which serve the chief purpose of supplying talking current to the calling subscriber from his own office, while still enabling him to send impulses to the distant office to control the switches. 
The second selectors in each office have the duty of picking out the desired thousand group and of selecting an idle third selector in that thousand. The third selector chooses the hundred group and the connector makes the final connection to the line of the called subscriber. In practice the connector switches are mounted on the same frame with the line switches with whose lines they connect. Incoming trunks, $d$, from other offices are wired to second selectors whose banks connect with trunks common to the local apparatus.

The means by which a subscriber controls the switches is

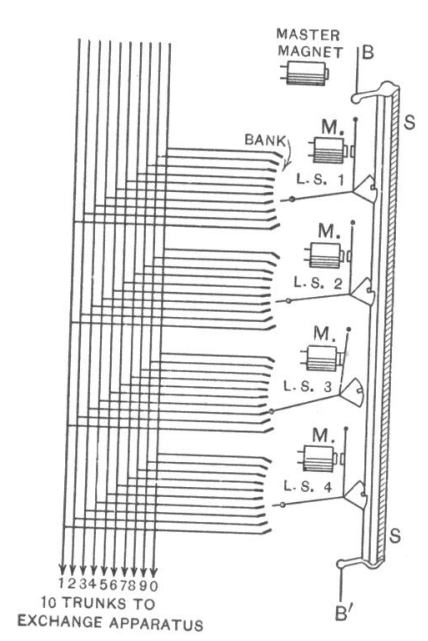

FIG. 3-Grouping of line switches

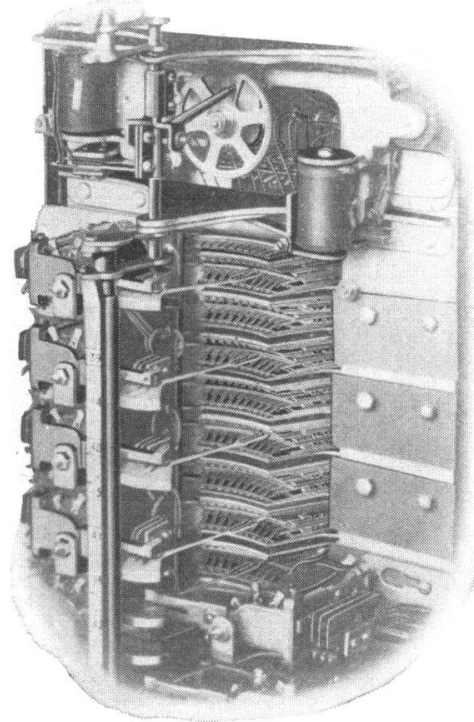

Fig. 4-Close view of line switch unit

briefly reviewed here to make clearer the operation of the special functions to be described later. The two line wires which extend from any Oakland office to a subscriber's station are termed "vertical" and "rotary" respectively. In the office each is connected to a relay which has its other terminal attached to a battery of storage cells. The other (positive) terminal of the battery is grounded. The dial or calling device at the substation, when operated, grounds each wire in a definite way, operating the relays, and through them the switches.

The vertical wire is the impulse transmitting member, for 
over it are sent at various times the exact number of impulses required to set the switches according to the digit called. The rotary wire is the switching or circuit-changing member. It determines upon what switch or magnet the vertical impulses shall act. At the close of any series of impulses over the vertical wire, one impulse is always sent over the rotary wire to shift the connections in the switches so that the next series over the vertical line will be effective on the next operation to be performed.

When the called subscriber answers, a relay in the connector is operated which switches the rotary wire from negative to

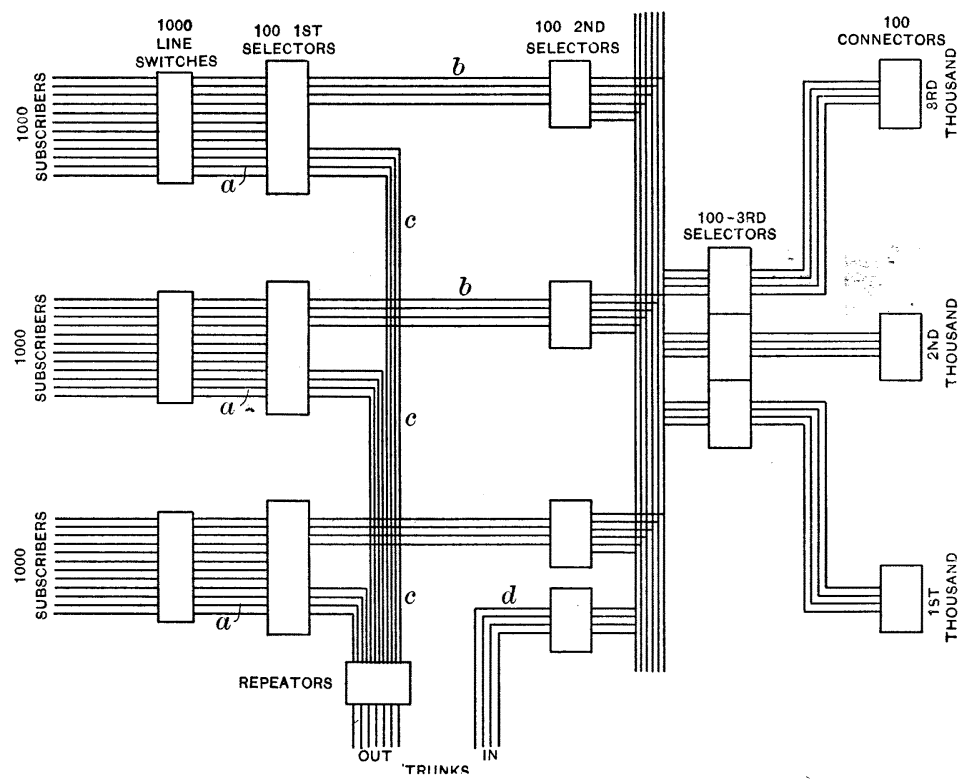

FIG. 5-Trunking in Oakland

positive battery, thus supplying talking current to the calling subscriber.

The simultaneous grounding and clearing of both vertical and rotary lines causes the switches to be released and restored to normal position.

San Francisco. The relative locations of the four offices are shown in Fig. 1. The grouping of switches is somewhat different from that of Oakland owing to the introduction of secondary line switches. A typical arrangement is shown in Fig. 6.

Each subscriber's line terminates in a primary line switch. 
These switches are grouped in units of 50 each, and have access to ten trunks, as described for Oakland. But here the difference begins. The trunks from the primary line switches are carried to secondary line-switch units, where each trunk terminates in a secondary line switch. Each unit group of secondary line switches has access to a group of ten trunks, $d$, each of which ends in a first selector. These secondary line switches are exactly like the primary switches in function, for they pre-select the idle trunks in exactly the same way.

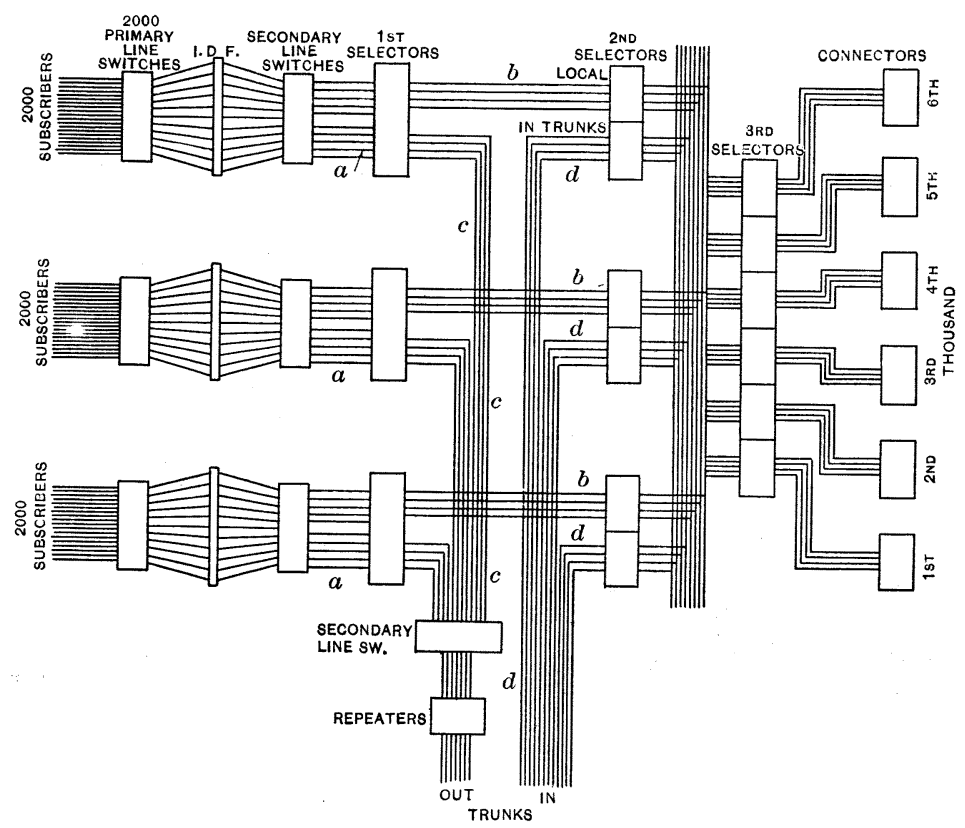

FIG. 6-Trunking in San Francisco

The purpose of using primary and secondary line switches is to enable any subscriber's line to use any first selector switch. This is in the interest of economy. Where only a small number of trunks are available for selection, the efficiency is relatively low. For instance, it has been found in automatic telephone work that ten trunks in one group can be depended upon to handle about 225 busy-hour calls, or 22.5 calls per trunk. Twenty trunks in one group will handle about 575 busy-hour calls or 28.75 calls per trunk. By arranging one hundred first selectors in one group 4,000 busy-hour calls may be successfully handled. 
If arranged in groups of ten each it would take about 180 first selectors to carry the same load.

The intermediate distributing frame placed between the primary and secondary line switches is to render more easy the interconnections by means of which the traffic is equalized on all the first selectors.

Since the first selectors select the office in which the called number is located, all levels but one will be connected to trunks to other offices. These outgoing trunks, $c$, are led through secondary line switches so that any inter-office trunk is made available to any first selector. For instance, if we consider a call from Main to South, every trunk leading to the South office from Main will be available for use by every first selector in the latter office. By arranging all the trunks in a common group, considerable saving in trunk cable is secured. Though varying with the local conditions, the saving has in certain cases been as high as 40 per cent.

The incoming trunks, $d$, from other offices terminate in second selectors which have access, in common with local second selectors, to third selectors. The grouping from here on is identical, in general, with that of the Oakland Exchange, and the connectors are mounted on the same frames with the primary line switches, whose lines they are designed to reach.

The exact method of operation differs radically from that used in Oakland, for the San Francisco apparatus is controlled by the subscriber over two wires with no earth return. This greatly sinplifies the subscriber's telephone and gives many structural and operating advantages.

The two subscriber's line wires are known as positive and negative, indicating the terminal of the battery to which each is attached through the windings of the line relay. The series of impulses for stepping up the wipers of the switches is caused by the dial or calling device, which opens and closes a normally closed pair of contacts. The line relay follows these impulses and repeats them to the magnets which move the wiper shaft.

The switching of the circuit to the next switch or operation depends upon the time interval between one series of impulses and the next. During a series, the circuit-changing member is held by a catch, which is released when the impulses cease to come. The release is accomplished by simply opening the line circuit by hanging up the receiver. This momentarily brings into action the release magnets which restore all switches to normal. 
1. Telephone Transmission. The nature of the completed circuit between two subscribers has a vital effect on the quality and loudness of transmission. Freedom from external disturbances is to be secured by properly transposed wires and perfect electrical balance. The former is secured by a large use of telephone cable, the latter by high insulation and properly designed apparatus. In the telephone switchboards installed in San Francisco and Oakland every talking circuit is balanced from one end to the other. Fig. 7 gives in simplified form the transmission circuit between two subscribers in separate offices. From the calling telephone to the called telephone there are four "bridges" or shunt paths. Each of these is a relay or a pair of relays, all of which are highly inductive, so that little loss is occasioned to telephone current. The impedance of each path from line to earth is made as nearly as possible equal to that of the mate. No extra coils are bridged to one side of the

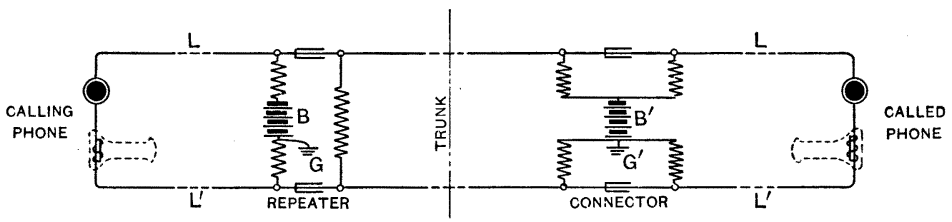

FIG. 7-Simplified talking circuit. Inter-office

line, and none are inserted in series. All auxiliary circuits are handled by a third wire within each office, leaving the talking circuit clear for its proper functions. The trunks are two-wire with no earth return circuits during conversation. Each subscriber is supplied with talking current from a battery in his own office. No repeating coils are used in any portion of the circuits.

2. Method of Calling. The method of calling a number on the automatic system has been described by others. Briefly, it consists in rotating a small dial by the finger, making one motion for each digit in the call number. The dial used in San Francisco is shown in Fig. 8. To assist the memory, the offices are designated by letters instead of figures. The code is as follows:

C Main office.

$\mathrm{J}$ Howard office.

M South office.

S West office.

Thus, a telephone whose number is 22785 , appears in the 
directory as J-2785. It is served by the Howard office, though this is of little importance to the subscriber.

The manipulation of the dial for an average call number takes about five seconds, after which the bell of the called station begins to ring without further action on the part of the calling subscriber. The ring is intermittent and ceases when the called subscriber answers.

Instantaneous release of the switches is effected by hanging the receiver on the hook. This is of interest chiefly to those who have two or more calls to make in succession.

3. Diversity of Languages. The method of calling above described is of special convenience to the many people who speak only foreign languages. The Arabic numerals are common to practically all nationalities represented in San Francisco except

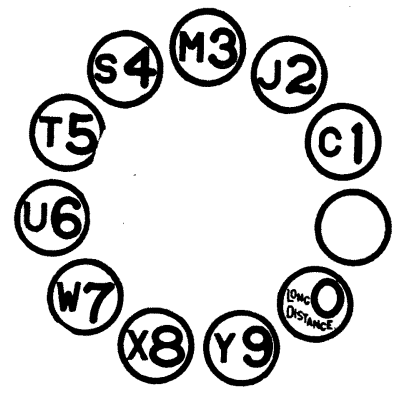

Frg. 8-San Francisco dial

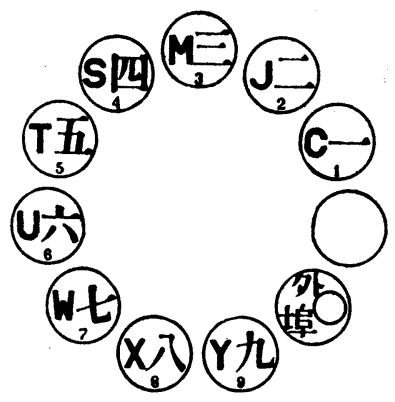

Fig. 9-Chinese dial

the Chinese. For the latter a special lettering has been made which is shown in Fig 9.

4. Measured Service. One of the conditions met in managing a telephone system in a large city is the desirability of charging for the service in proportion to the amount of service rendered. This is ordinarily done by attaching a meter to each subscriber's line. The requirements of a meter are as follows:

1. It must register only once for each completed connection, rejecting those in which the called station fails to answer.

2. It must make no charge for connection to certain classes of telephones, such as fire, police, information and complaint desks, etc.

The Oakland exchange is operated on the flat rate basis for all calls originating and terminating within the exchange, which includes the suburb of Berkeley. In San Francisco measured service is required. 
The meter in an automatic telephone system is attached to the line switch. As above mentioned, it must not operate until the called station answers. Therefore, the latter act must cause some change in electrical condition at the line switch which will cause the meter to register. The line switch of the calling station and the connector which picks up the called line may be in different offices with 1st, 2 nd, and 3 rd selectors and a repeater in the circuit between. The called station can control the connector and through it the condition of the line leading back to the line switch. Two changes in condition are available, reversal of current and change of current strength. The latter is undesirable on account of its interfering with conversation to stations set aside for free service. The former seemingly necessitates some form of polarized magnet. As it was desired to avoid the use of permanent magnets, a two-coil meter was devised. It is so arranged that when the called station answers, the current supplied to the latter operates the relay which reverses the current supplied to the calling line. This causes the meter to record one call. Neither coil alone will cause registration, and the apparatus has a range from 0 to $1,500 \mathrm{ohms}$ line resistance. Its line coil is of low resistance and is short-circuited during conversation. The reversal of current for operating the meter is accomplished in such a manner as not to cause inconveniences to the calling subscriber.

5. Free Service. Since the connector is the switch in which occurs the reversal of current which operates the meter, the means for giving free service must effect this part of the apparatus. It is done by grouping all free service lines such as information, police, etc, into one or more hundred groups, each group served by a set of connectors. All these connectors are so wired that the current flow to the calling subscriber is not reversed when the called station answers, consequently no register is made.

There is one class of calls for which, strictly speaking, no charge should be made, and that is calls which result in the wrong number being obtained. The meter makes no discrimination here, and if the called station answers, a call will be recorded. However, experience has shown that such a small per cent of wrong numbers are obtained that they may safely be neglected. This is especially true if wrong calls due to carelessness in the use of the dial be omitted. For these it is right to expect the subscriber to pay. The same is true of regular manual practice, for if a subscriber calls for the wrong number and gets it, he is expected to pay for it. 
Fig. 10 is a general view of a number of primary line switches. In the space above the switches indicated by the letter $M$ the meters may be seen. These are in tight cases which are locked and accessible only to the official who has charge of meter reading.

Cash measured service is furnished by means of coin boxes attached to the line wires at the subscriber's stations. No coin is required to be deposited in order to call. When the called station answers, the reversal of current operates a magnet in the

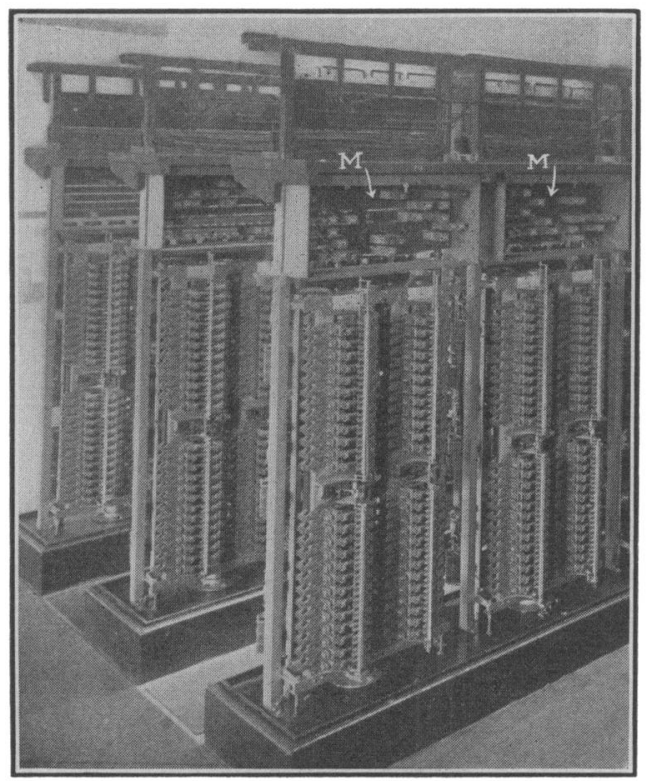

FIG, 10-Primary line switches with meters

coin box which short circuits the transmitter and places a shunt in paraliel with the receiver. This prevents the transmission of speech, but allows the user to hear faintly the voice of the called subscriber. By dropping a coin in the chute, the shunts are removed, so that conversation can take place.

When a call is made to a free station as above described, no reversal of current takes place, so that the calling subscriber is not forced to make a payment.

6. Private Branch Exchanges. In both Oakland and San Francisco the nature of the business makes quite a large number 
of private branch exchanges profitable to the subscriber and to the operating company. These boards are manually operated and have several trunks connecting them with the nearest public exchange office. Each business house is listed under one number in the directory. When a public subscriber selects the call number the connector switch must automatically select an idle trunk to the private branch board. This is done by a special type of switch known as the rotary connector. It responds to the impulses for the tens and units digits of the call number, but immediately thereafter acts as a selector until an idle trunk is found. These rotary connectors are grouped in hundreds so that in providing for this special service the call numbers are set aside in blocks of one hundred each. Thus in the Main office San Francisco, the 1100, 1200, 2100, 2200, 3100, 3200,4100 , and 4200 hundred units are set aside for private branch exchange service and no number in them assigned for individual lines.

Let us suppose that the call number of a certain wholesale house is $\mathrm{C}-4251$ and that four trunks are required for the business. The connector switches in the 4200 group of the Main or C office will serve this board. These four trunks will be multipled to the first four sets of contacts in the fifth level of the connector banks, that is, corresponding to numbers 4251,4252 , 4253 , and 4254 . Contacts 4255 are connected to the busy tone so that if it happens that all trunks are occupied, the calling subscriber will receive the busy signal and be induced to release. The private contacts 4255 of the several connector switches are not multipled together. If while all four trunks are occupied, more than one called is received for the busy number, the second and later calls will also stop on 4255 and not be forced past to 4256 which might be assigned to other service.

If desired, the remaining four numbers in this same level, $4256,4257,4258,4259$ may be given to some other private branch board. In fact any grouping of the trunks of a level may be used, it being only necessary to reserve one set of contacts at the end of each group for busy indication.

The trunks to private branch board are treated exactly like subscriber's lines in the public exchange, each being wired to a line switch for calls to the automatic system. They terminate in jacks and signals on the manual private boards and can be used for establishing connections either to or from the subscriber. The operator is provided with a calling device which may be 
switched by keys to any cord circuit. With this the operator calls into the automatic exchange and arrangements are made so that complete double supervision is secured on all calls.

In the case of a private branch exchange having sufficient magnitude of business to require it, the trunks leading from connector banks may be made one-way only and reserved for calls to the branch board. There will then be installed as many trunks from the latter to the public exchange as are necessary, these trunks being attached to line switches. The number of trunks is not limited by the number of contacts in a level of the connector bank.

7. Transbay and Suburban Toll. We come now to what is perhaps the most interesting feature of the combined telephone exchanges. On account of the relative positions of the two cities, and their close business and social relations, the matter of quick and satisfactory communication between them is of great importance.

The fact that a charge is made for all calls between the two cities makes it necessary to employ operators for putting up these connections, making records of the same.

Long distance toll work is necessarily handled in a different manner from suburban calls. In the former, the telephone company agrees to bring together two persons, in the latter merely two telephones. This is sometimes called the "two number basis" inasmuch as only the two numbers are recorded, no attention being paid to names.

A common method of handling suburban as well as long distance calls is to have each call received by a recording operator, who makes out a ticket. She tells the subscriber that he will be called when the line is ready. The ticket is then passed to a line operator, who calls the distant city and with the coöperation of a similar operator there, establishes the desired circuit. She then calls the station whose number was given by the person originating the call and allows the conversation to take place.

For the suburban work between San Francisco and Oakland it was thought best to abolish this slow procedure and use what is known as the "rapid fire" method. Accordingly provisions were made for allowing the recording operator at San Francisco, for example, to complete the call into the Oakland exchange by a calling device in San Francisco, and to allow the conversation to proceed at once. This virtually makes the recording operator a line operator and entirely dispenses with the services of a line operator to complete the connection in the distant office. 
The recording operator is of course obliged to ask the subscriber who originates the call for his telephone number so that the cost of the toll service may be properly charged. It is to be expected that, either by accident or intention, wrong numbers will sometimes be given. To prevent error from this source a system of back checking has been devised, which will be described in detail later.

The operators are divided into two main groups, suburban and long distance. The latter are as usual made up of recording operators and line operators, each with the usual duties assigned.

The suburban operators work in pairs or threes and are termed suburban operators and checking operators. All calls for out of town come to the suburban operators, who act as distributors between the two boards. This is because of the relatively heavy nature of transbay and suburban service as compared to long distance service. The suburban operator puts up suburban connections while the checking operator verifies the correctness of the number given by the calling subscriber. Usually one checking operator verifies the work for two suburban operators, though during periods of light load the latter check for themselves.

The method adopted in making a call is as follows: The subscriber takes his receiver from the hook and calls the digit " 0 " on the dial. This lifts the wipers of a first selector to the top level and selects an idle trunk to the suburban board. Here a lamp attracts the attention of a suburban operator who answers the call by plugging into the jack and throwing the listening key on her cord circuit. She asks whether " suburban" or "long distance" is wanted. If the latter, she momentarily depresses the transfer key button associated with the trunk in use. This automatically transfers the call to the long distance recording board, where multiples of the incoming trunks appear. The proper operator, seeing the burning lamp, answers and cares for the call in the usual way.

If, however, the subscriber replies that he wants "Suburban ", the suburban operator asks him two questions.

"What number do you wish?"

"What is your number?"

This information she writes on a ticket together with the number of the incoming trunk over which the order was received. The pad of tickets bears the suburban operator's position number and is arranged to furnish duplicate copies. Having recorded the information she passes the duplicate ticket to the 
checking operator who is seated directly in front of her. The two following operations are then performed simultaneously:

The suburban operator selects an idle outgoing trunk leading across the bay and calls the number in the distant exchange by means of her dial. The checking operator upon receiving the ticket plugs into the multiple of the incoming trunk shown thereon. Then by an independent set of apparatus she calls the number of the originating subscriber. If the checking operator finds that the number written on the duplicate ticket is wrong, she passes the ticket back to the suburban operator by dropping it in a special ticket box in plain view. The latter operator at once throws the cut-off key on the cord, thereby severing the connection from the outgoing transbay trunk. She then requests the calling subscriber to repeat his number. If he repeats the number previously given, she informs him that she can not reach him over that number. In most cases the subscriber now corrects his mistake by giving the true number. This is now entered on the tickets and the duplicate again passed to the checking operator for verification. Usually the suburban operator allows the connection to proceed, while the second check is being made, for, as a rule, the second number is found to be correct.

On the suburban operators cord circuit is provided two supervisory lamps which have the usual functions. The calling supervisory lamp burns till the subscriber in the distant office answers. Then the operator stamps the ticket in a calculagraph. At the end of the conversation both subscribers hang up the receivers. This lights both supervisory lamps. The operator then times the ticket and disconnects the circuits.

The completed circuit over which conversation is carried on is shown in simplified form in Fig. 11. It is balanced and quiet in service. The rapid break and make of the calling device in operating the switches through the cable under the bay causes no inconvenience to those using parallel trunks.

When a subscriber has secured connection with the suburban or toll board, it is necessary that he be permitted to signal the operator by moving his receiver hook up and down. This would ordinarily cause the release of his first selector since all automatic switches are arranged to be released by the depressing of the hook lever. To prevent this breaking of the connection a special arrangement is attached to the outgoing toll trunks from each office in place of the regular repeater. This enables 
the release of the first selector used by the subscriber to be controlled by the operator.

After the subscriber has hung up, the operator pulls out her plug which automatically causes the release of the first selector.

8. Toll Checking Apparatus. In the preceding general description of the mode of operation, reference was made to the toll-checking operator and her apparatus. The general plan of checking is to connect to the incoming toll trunk in use a source of alternating current which produces a musical tone. By independent automatic switches the checking operator calls the number given. If correct she will obtain connection to the

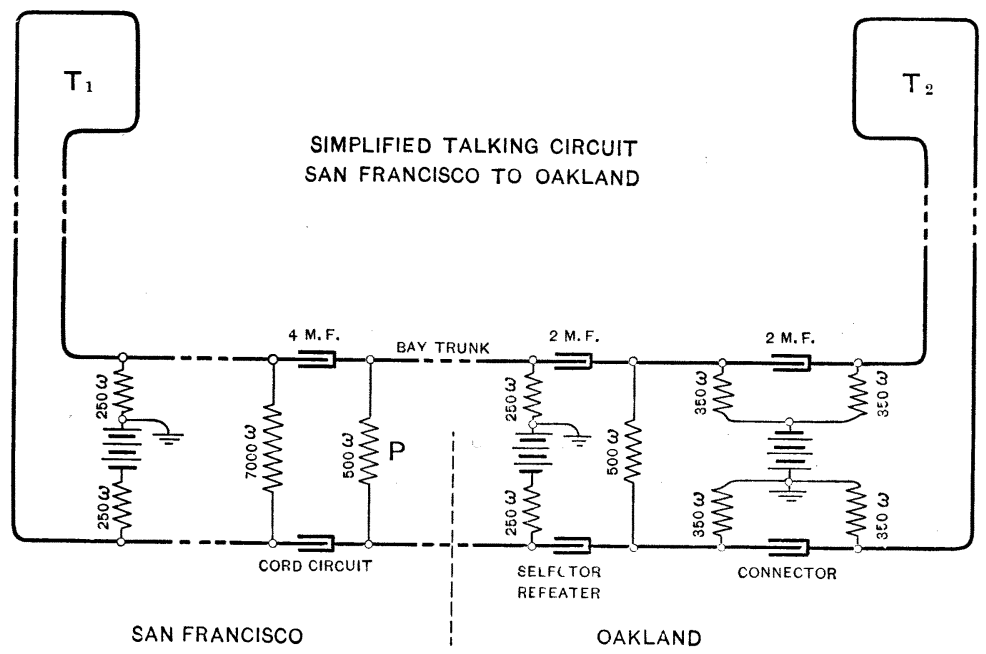

FIG. 11-Simplified transbay talking circuit

same line as that over which the call was received by the suburban operator, and by listening can hear the tone. The condition is shown in Fig. 12. The subscriber at the right has called the suburban operator over the incoming toll trunk. The operator has answered and made out the ticket. The checking operator has used a checking trunk to secure connection with the number given. At this point she presses the check tone key which connects to the incoming toll trunk a source of alternating current which produces a sound differing from the regular busy tone of the exchange. If the number is correct, the tone will be carried back over the toll trunk to the connector multiple and thence over the checking trunk to the checking operator. 
The ordinary connector cannot be used by the checking operator in picking up the calling subscriber's line, because the latter is protected by a busy test which will not permit an ordinary connector to establish connection. For this work one check connector is provided in each hundred group. It is devoted exclusively to testing and checking. It is provided with three pairs of wires, one pair $(A$ and $B)$ for carrying the tone, another pair $(+$ and -$)$ for operating the magnets for lifting and rotating the wipers and a third pair for auxiliary purposes.

The checking operator secures connection with the check connector in any hundred in any office by a system of first, second and third selectors which is entirely separate from the regular apparatus of the exchange. One first selector repeater is pro-

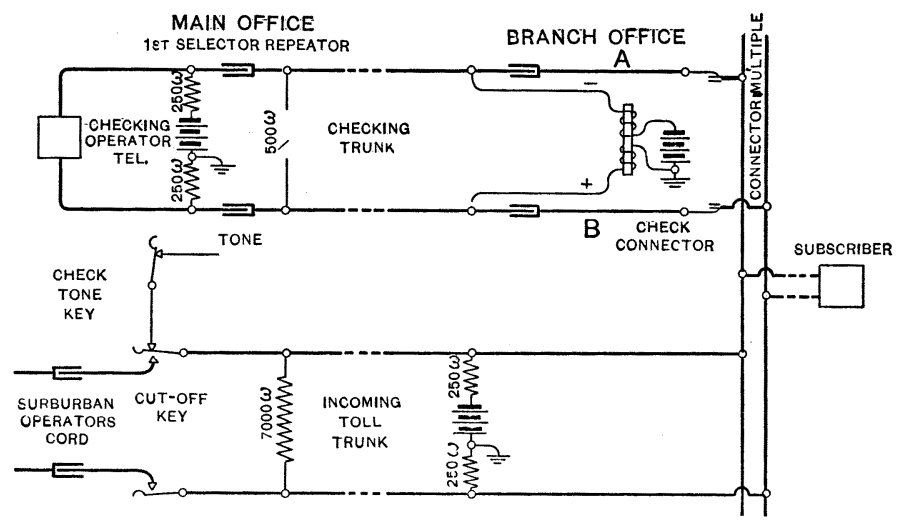

FIG. 12-Simplified toll checking circuit

vided for each checking operator, to enable her to select a trunk to any office in the exchange. In each office as many second selectors and third selectors are provided as are necessary for the work, the number varying in the different offices. These are operated by a calling device located at the checking operator's position.

When the checking operator plugs into a connection it lights a red lamp associated with the suburban operator's cord circuit. This shows that the checking operator has picked up the trunk. When the checking operator has verified the number and withdrawn her plug the red lamp is extinguished, thus notifying the suburban operator that the number has been checked and is correct. 
As a guard, tending to prevent the checking of wrong trunks, the checking operator's cord circuit is provided with a lamp. This lamp will light when plugged in on a trunk which is waiting for the complet on of a connection. If, however, the checking operator by mistake plugs in on a non-busy trunk, or one over which a connection has been completely established the lamp will not burn.

9. Credit and Cash Tolls. The larger proportion of the calls between the two cities are handled on the credit basis. From the records of the toll tickets, statements are made out at regular intervals and sent to the subscribers for payment. For such public places at which it is desirable to collect cash for suburban and long distance service, a regular coin box is attached to the

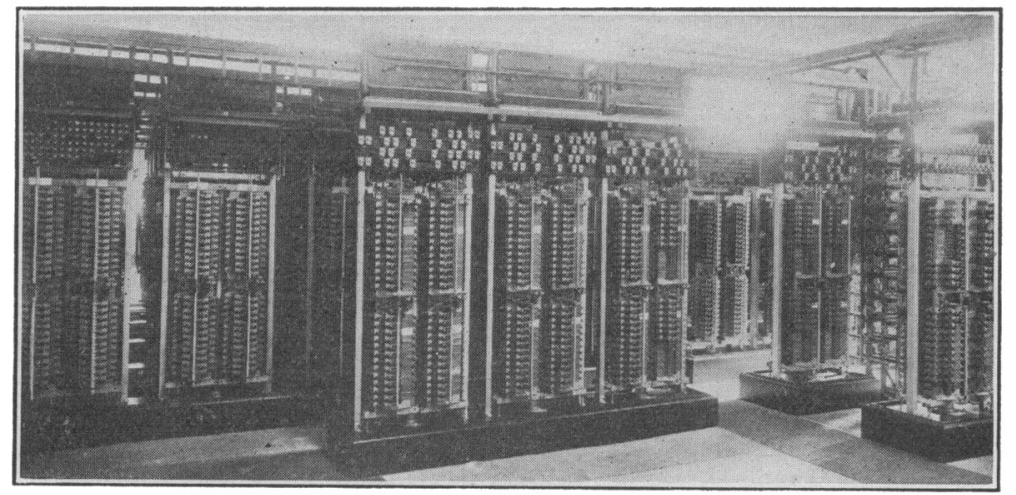

FIG. 13-Secondary line switches

telephone. The dropping of the coin gives a signal which reveals its denomination to the operator.

10. Two Three-wire Operation. In the general description of suburban toll operation a short reference was made to the trunk lines connecting the two cities. For the purpose of giving a better idea of the arrangement, a trunk line will be described which handles calls from San Francisco to Oakland.

The work of interconnecting the two exchanges was rendered more difficult by the fact that the Oakland apparatus is threewire, while that of San Francisco is two-wire. The two-wire system requires only the opening and closing of the line circuit. The three-wire system requires each line wire to be grounded in a certain order. The San Francisco recording operator using 
a two-wire calling device was required to operate three-wire selectors and connectors in Oakland, in some cases as far as thirteen miles away. This was done by means of repeaters which handle the impulses somewhat like a telegraph-repeater, except that it is necessary to transmit impulses only one way.

Each transbay trunk from San Francisco to Oakland ends in multiple jacks with visual busy signals on the San Francisco suburban board, and in a first selector-repeater in the Main office in Oakland. This switch is a first selector, to pick out trunks to the desired office in Oakland, combined with a repeater arrangement to convert the simple make and break of the twowire system into the alternate grounding of vertical and rotary lines required by the three-wire system. The release of the connection as well as proper supervision is also provided in the repeater.

The operator is provided with a set of plugs and cords with which to connect incoming trunks from San Francisco subscribers to outgoing trunks to Oakland. A calling device is mounted on the keyboard and arranged to be switched into any cord circuit by keys. When a call comes in over an incoming trunk, it is answered by inserting the answering plug of a cord into the proper jack. An idle outgoing trunk to Oakland is selected by inspection of the visual busy signals, and the calling plug of the cord in use inserted into the jack. The calling device is then switched into the cord circuit and rotated in accordance with the desired number. This operates the switches in Oakland.

The supervision is accomplished as follows: Across each cord circuit of the suburban operator in San Francisco is bridged a polarized relay, $P$, Fig. 11 . Normally the current from the first selector repeater in Oakland is in such a direction as to cause the relay to light its associated lamp. In the Oakland exchange the circuits from the first selector through to the connector are three-wire. When the called subscriber answers, it causes the rotary line to be switched from negative battery to ground or positive battery. This energizes a pair of relays in the first selector repeater which reverses the current supplied by the latter to the operator's cord circuit in San Francisco. This reversal causes the bridged polarized relay $P$ to operate, extinguishing the supervisory lamp.

The release of the three-wire Oakland apparatus calls for the momentary simultanenus grounding of the vertical and rotary 
lines. The two-wire first selector repeater at the Oakland end of the transbay trunk requires only the simple opening of its line circuit By a suitable arrangement this is made to cause the release of the three-wire apparatus according to the manner just indicated.

The trunks from Oakland to San Francisco are operated straight two-wire, and hence are simpler than those just described. For the sake of securing sharper, better signals the trunks are wired through the first selector repeaters in the San Francisco main office. Supervision is secured by marginal relays. 